\title{
Living robots
}

T he original 'robots', described in the 1921 play R.U.R. by the Czech writer Karel Čapek (the word is Czech for 'labourer') were not made from steel and controlled by electronics, but were fleshy and autonomous. Čapek's manufacturing process, in which organs and other parts were made from vats of fleshlike dough and assembled into bodies, took inspiration from the emerging technology of in vivo tissue culture. It blurred the boundaries between engineering and biotechnology in a way that seemed far beyond the technologies of the time.

The results now reported by Kriegman et al. make this vision seem almost unnervingly prescient ${ }^{1}$. They describe 'reconfigurable organisms' made from living cells assembled into conglomerates about a millimetre across with arbitrary shapes, which are designed in silico for particular functions such as locomotion. These structures have been dubbed xenobots - which might be given the literal and apt interpretation of 'strange robots', although here 'xeno' comes from the use of embryonic stem cells of the African clawed frog Xenopus laevis as the construction material.

The cells are harvested from embryos at the blastula stage, when they mostly retain pluripotency: the ability to grow into any tissue type of the body. But particular functions are engineered into the design by using cell types that have begun to differentiate towards a target tissue. For example, passive tissue may come from the progenitors of epithelial cells, which line the surfaces of organs, blood vessels and skin. Motile tissues for movement, meanwhile, can be constructed from cardiac progenitor cells, the precursors to heart muscle, which contracts by supporting waves of electrical activity.
The researchers cultured these cell types separately and then pooled them in the desired numbers so that they could aggregate into a single mass. The specific arrangement of cells was decided by using an evolutionary algorithm in simulations that seek the geometries that performed best at the target task. It was then engineered by hand with a combination of microsurgery forceps to manipulate cells and a fine cautery electrode to remove them at certain locations. The resulting cellular structures are necessarily rather crude approximations of the original design, but Kriegman et al. show that they can work as intended. For example, a 'two-legged xenobot' with contractile cells on its lower half was able to show non-random, directional movement over a surface.

The designs can be optimized by iteration, using the best performing structures as the input for further rounds of in silico evolution: there will surely be an empirical element to such a process. The motile structures also showed emergent behaviours, both in silico and in vivo, for example temporarily attaching to or orbiting one another when they collide. Some evolved designs included unexpected features that the researchers say could be opportunistically 'exapted' for new uses. One 'walker' evolved a hole in the design stage to reduce hydrodynamic drag, which might function instead as a cavity for storing and transporting objects.

Kriegman et al. speculate that 'living' structures like these might provide biocompatible devices for in vivo drug delivery or tissue repair. The limitations are yet to be fully explored. Cells in natural tissues, for example, tend to fill in concavities spontaneously, restricting the stable geometries. And stem cells have their own agendas: their fates

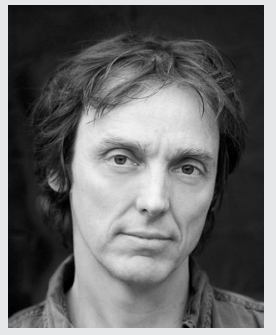

Philip Ball

are governed by the signals received from surrounding cells, and some are themselves mobile in developing tissues, so that these xenobots, if made from cells not yet committed to a specific fate, might begin to subvert the original designs. Maybe this spontaneous development could itself become an empirically guided design element.

Such autonomy raises a more profound question. Kriegman et al. call their structures, perhaps controversially, 'organisms'. But when does engineered tissue become organism? At present, they lack the reproductive capacity that might be deemed an essential feature of a real organism (though some are of course by their nature sterile). It's by no means obvious that they could not be given that ability - with attendant biosafety and ethical considerations. They might even be equipped with simple cognition too. We would then need to ask: are multicelled aggregates plastic enough to support totally different yet wholly viable lifeforms from the ones their genomes have evolved to create?

Published online: 25 February 2020 https://doi.org/10.1038/s41563-020-0627-6
References
1. Kriegman, S., Blackiston, D., Levin, M. \& Bongard, J. Proc. Natl Acad. Sci. USA 117, 1853-1859 (2020). 